\title{
A Framework for Entry \\ PAR values and engagement strategies in community research
}

Gateways: International Journal of Community Research and Engagement Vol 3 (2010): X-XX CUTSePress and the authors

ISSN 1836-3393

\section{Joanna Ochocka \\ Elin Moorlag \\ Rich Janzen}

Centre for Community Based Research

The purpose of this article is to explore and clarify the importance of entry in community-based research on sensitive topics such as mental health and to suggest a framework for community research entry that uses the values of participatory action research (PAR) and specific engagement strategies. We argue that the entry process is a critical aspect of the overall research process. Indeed, its success or failure sets the tone for the entire project.

We wish to emphasise three points about research entry. First, that successful research entry is not only the effort of recruiting participants and/or gaining access to communities at the beginning of the research study, but is an ongoing and gradual process of community engagement that involves much work (before and throughout all phases of research). Second, that to be successful, research entry requires the building and maintenance of long-term and reciprocal relationships between researchers and community members with power imbalances to be constantly navigated. Third, that research entry should be guided by a combination of PAR values and concrete engagement strategies. To this end, we offer a discussion of our understanding of entry and the PAR values that inform it, as well as a critical evaluation of our own case study, examining strategies employed and challenges faced. While we consider the principles of entry discussed in this article to be relevant throughout the entire research process, the article looks primarily at entry processes at the initial stages of research.

This article draws from a case study of a collaborative community-university research project completed in the Waterloo and Toronto regions of Ontario, Canada. The 'Taking Culture Seriously in Community Mental Health' is a Community University Research Alliance (CURA) project involving over 45 partners who explored concepts of mental health and mental illness as well as needed services and supports from five cultural-linguistic perspectives (Somali, Sikh Punjabi, Polish, Mandarin, Spanish Latin American). A primary aim of the research was to inform new demonstration projects and the future organisation and delivery 
of mental health services in multicultural Canada. There are a limited number of case studies with mental health consumer/ survivor organisations that have explored the use of PAR values in community-based research and entry (Nelson et al. 2004, 2010; Nelson, Lord \& Ochocka 2001), and we believe that a similar approach can be helpful in researching sensitive topics with culturally diverse communities.

\section{COMMUNITY-BASED RESEARCH: ISSUES REGARDING ENTRY}

For this article we use a definition of community-based research developed in 2009 by 25 community and academic researchers who came together to address community-based research ethics in the Waterloo Region, Canada. Drawing inspiration from Israel, Schulz, Parker and Becker (1998), community-based research was defined as research that strives to be:

- community situated - beginning with a research topic of practical relevance to the community (as opposed to individual scholars) and which is carried out in community settings

-collaborative - community members and researchers share control of the research agenda through active and reciprocal involvement in the research design, implementation and dissemination - action-oriented - the process and results are useful to community members in making positive social change and promoting social equity (for more information, see: www.communitybasedresearch. ca/Page/View/CBR definition.html).

In community-based research the relationship between researcher(s) and participating community members is vital to the research outcome and is always, at the same time, under negotiation. The process that researchers undertake in order to gain entry into communities is a central element of the entire project, yet surprisingly, the topic of entry is rarely addressed in the relevant literature and is an aspect often overlooked within community-researcher partnerships. Overwhelmingly, articles outlining qualitative approaches to community-based research in the social sciences, even those involving sensitive issues, gloss over the entry process and instead focus on strategies for participant recruitment (Parrado, McQuiston \& Flippen 2005; Sadavoy, Meier \& Ong 2004; Sixsmith, Boneham \& Goldring 2003).

This is perhaps understandable when considering research from a traditional perspective, where it is often viewed as a tool for gathering and accumulating data (Babbie 1998). From this standpoint, research entry is seen as a means for recruitment or research access. Yet if research is viewed as intervention, as social action with the potential for change, then entry becomes the critical opportunity for establishing a community-researcher relationship with markedly different dynamics and goals in mind. Crucial to this understanding is a conception of entry as a process, one that is shaped by a combination of participatory action research values 


\section{3 | Gateways | Ochocka, Moorlag \& Janzen}

and concrete engagement strategies. In this scenario, participants and researchers alike have access to the processes as much as the outcomes, and the research itself is a means for change.

Building from experiences gained from our own projects with community-based participatory research, we propose that entry is a vital and integral component of the research process, and thus the entry strategies or techniques used must be carefully considered and respectfully executed. Entry becomes the gateway to successfully establishing community-researcher collaboration.

Despite an apparent need for the notion of entry to be addressed as a distinct element in the research process, an insubstantial body of literature focusing on community entry would suggest the issue is frequently neglected. There are, however, a handful of sources (Lee included) that provide insight along with tentative guidelines for successful community entry. Points of convergence among these sources occur around the following topics: engaging community gatekeepers; emphasising collaboration and involvement of community stakeholders in the project; maintaining a presence in the community; and being attentive to initial hesitancies and the 'politics of distrust' (Lee 1993). More specifically, presented by Tareen and Omar (1997), are a series of five steps that might be taken by participatory researchers in order for community entry to be accomplished. These steps include drawing community members in through open discussion and analysis of research intentions and strategies; inviting stakeholders to be project collaborators; and encouraging community members to take the lead on some components of ongoing action. Further discussion is provided by Sixsmith, Boneham and Goldring (2003) on the significant role of community gatekeepers and some of the complications that may arise in the presence of multiple gatekeepers with conflicting views, or restrictive gatekeepers who deny researchers access to the community.

For us, community research entry is a process to establish a long-term relationship with community members that is 'continually negotiated' (Sixsmith, Boneham \& Goldring 2003), with power imbalances to be constantly navigated. Successful entry also can be a method for increasing participation and accessing under-researched populations. In this article we suggest four distinctive research entry stages:

- pre-engagement

- engagement

-assessment, reflection and feedback

-ongoing maintenance.

At each stage, entry strategies and mechanisms need to be rooted in the values of PAR.

We agree with Lee (1993) that in social research, progressive entry is needed, whereby the researcher attempts to minimize the social distance between themselves and the participants through frequent contact (the ethnographic approach of being there and 
being seen [Sixsmith, Boneham \& Goldring 2003]), and by making requests for access that gradually increase. We also agree with Minkler (2004), who highlights that the insider-outsider tensions that arise when conducting community-based participatory research often are the result of negative historical relationships with institutionalised and internalised oppression.

The question of why the entry process has been so seldom recounted, explored and theorised is pondered and discussed by Lee (1993) in a thorough overview of the process of access in research on sensitive topics. Possible suggestions include time limitations as part of the research-to-publication expectations; the one-sided nature of such narratives, which would potentially give only half of the picture; and a vague or even a lack of knowledge by the researcher as to the reasons behind successful entry (Lee 1993). A further possible reason added by Lindsay (2005) is that this oversight is embedded within the current presentation structure for research and findings in academic journals. The dominant standard for what should and should not be included generally discourages researchers from addressing and discussing the issue of entry.

\section{PARTICIPATORY ACTION RESEARCH: A VALUE-DRIVEN APPROACH TO RESEARCH}

Participatory action research (PAR) can be defined as 'a research approach that consists of the maximum participation of stakeholders, those whose lives are affected by the problem under study, in the systematic collection and analysis of information for the purpose of taking action and making change' (Nelson et al. 1998, p. 12). PAR can be characterised as 'research with', not 'research on' people. It provides training and mentoring for members of the community so that they can learn how to research; offers opportunities for meaningful involvement in a project that is intended to effect community change; produces data for advocacy; and places a high value on experiential knowledge (Ochocka 2007; Ochocka, Nelson \& Janzen 2005).

The PAR approach is rooted in the ideals of democracy, equality, liberation and change (Nelson et al. 2004; Ochocka 2007). It includes a commitment to power sharing, relationship building, and a shared ownership and control of research. PAR researchers are therefore value-driven and use values to evaluate the success of their research projects.

There are four main values that underlie PAR and influence all components of the research process: empowerment; supportive relationships; social justice; and ongoing reciprocal education (Nelson et al. 1998; Ochocka \& Janzen 2007). We want to suggest adding a fifth value: respect for diversity, which emphasises equity and inclusion. A discussion of these five PAR values follows.

\section{Empowerment}

The first PAR value focuses on developing personal and/or group power from a process of working together towards a common 
goal. Empowerment is a transitive process between the individual and the environment around him/her (Zimmerman 1995) with some transforming results in power sharing (Rappaport 1981). It is based on individual and community self-determination and involves individuals (or groups) interacting together and gaining power to make choices and transform them into desired actions and outcomes (Nelson, Lord \& Ochocka 2001). Empowerment also refers to personal changes, resulting in greater control, voice, skills, assertiveness and self-esteem (Chamberlin 1997; Zimmerman 1995) and to other benefits that come from true collaborations.

\section{Supportive Relationships}

The second PAR value deals with the type of relationships built between researchers and community members. In particular, this value addresses potential inequalities in the research relationship. Supportive relationships foster a connectedness not only to the research topic area, but also to the people involved in the research processes (Nelson et al. 1998). By collaborating and sharing stakeholders' diverse knowledge and experience, community-based research seeks to change the social and personal dynamics of the research situation so that it is cooperative and enhances the lives of all those who participate (Stringer 2007).

\section{Social Justice}

The third value of PAR emphasises the practical implications from research - its social action agenda. Community-based research needs to produce useful knowledge to make positive social changes and to promote social equity. It strives to integrate research and practice, reduce the gap between knowledge and action and thereby create a new future that is better than the present (Kemmis \& McTaggart 2005; Schensul 2009).

\section{Ongoing Reciprocal Education}

From the perspective of PAR, reciprocal education refers to the value of mutual learning and mutual education. There is an understanding that knowledge is produced and disseminated in a meaningful way among researchers, community partners and others on an ongoing basis. Respect for knowledge inherent within communities is particularly important (Wenger, McDermott \& Snyder 2002). Such a notion breaks from the traditional assumption that researchers bring knowledge to communities. In fact, the practical and experiential knowledge of community members are seen to be integral to the research endeavour, as they bring a 'knowing in action' that goes beyond simple knowing about action (Heron \& Reason 1997; Reason 2006).

\section{Respect for Diversity: Equity and Inclusion}

We have added this value to the identified PAR values because community-based research is conducted in the real world where respect and appreciation of various perspectives, beliefs and norms are essential. This is especially so when researching with culturally diverse groups on sensitive topics. 
We believe that using the values of PAR as a guide for implementing research entry strategies is essential in facilitating the development of successful, long-term community-researcher relationships. These PAR values act as a framework for communityresearch entry. Further, such a value-driven approach enables community members to become partners and collaborators in the research endeavour.

\section{THE CURA PROJECT}

'Taking Culture Seriously in Community Mental Health' was a five-year (2005-2010) Community University Research Alliance (CURA) project, bringing together over 45 university and community partners in the Toronto and Waterloo regions of Ontario, Canada. The purpose of this research collaborative was to explore, develop, pilot and evaluate how to best provide community-based mental health services and supports that would be effective for people from culturally diverse backgrounds. This research study was led by the Centre for Community-based Research (CCBR), located in the neighbouring cities of Kitchener and Waterloo in Ontario. CCBR has a long history (since 1982) in conducting research that uses a PAR approach focusing on sensitive topics and involving marginalised communities (see www.communitybasedresearch.ca/).

Five cultural-linguistic communities were actively involved in this project (Somali, Sikh Punjabi, Polish, Mandarin Chinese and Spanish Latin American communities) at the two sites (Toronto and Waterloo; 10 groups in all). Some of these communities had previous research experience with the CCBR (for example, the Somali and Spanish Latin Americans) but researchers were approaching others for the first time. The cultural-linguistic groups were chosen based on the demographics across sites (both newer and established communities in Canada with sufficient numbers); geographic distribution of region of origin; differences in migration experiences (immigrants versus refugees, voluntary versus forced migration); and visible minority status. One of the project's goals was to emphasise the transferability of knowledge gained by migrants to all of multicultural Canada (see Janzen, Ochocka \& the 'Taking Culture Seriously' Partners 2006). All 10 groups worked collaboratively with each other and with other stakeholders including researchers, mental health practitioners, funders and policy makers.

The study had three phases, each using a PAR approach:

-Phase I: to explore diverse conceptualisations of mental health problems and practices based on the use of several mixed methods

-Phase II: to develop culturally effective demonstration projects based on study learnings

-Phase III: to evaluate demonstration projects.

The findings from the first phase of the research led to various understandings: of ethno-cultural community perspectives on mental health (Simich et al. 2009; Simich, Maiter 


\section{7 | Gateways | Ochocka, Moorlag \& Janzen}

\& Ochocka 2009); how to lead a large community-university partnership (Jacobson et al. 2007); and how to take culture seriously in community mental health and promote the wellbeing of immigrants from diverse cultural backgrounds (Janzen et al. 2010). The second phase of the research examined the planning of 12 proposals for CURA demonstration projects designed to meet the needs uncovered during the first phase (Nelson et al. 2010). The third phase focused on evaluating the implementation and outcomes of four of the CURA demonstration projects that were externally funded.

There were seven main mechanisms of engagement used to implement the PAR approach (Ochocka \& Janzen 2007). First, the CURA Partnership Group, which included representatives from all partner organisations, guided the study and made all strategic decisions. Second, a series of face-to-face site visits with ethnocultural community leaders were held so that they could help shape the project proposal; another round of visits was also held immediately after receiving funding. Third, local multi-stakeholder steering committees led the research component within each site. Fourth, researchers from the participating ethno-cultural communities were hired and trained as researchers and mobilisers of their respective communities. Fifth, ongoing communication and feedback was provided to research participants, community members and CURA partners, which included a number of forums for community members and two conferences to share findings and plan future activities within and outside the alliance. Sixth, 12 innovative concrete projects were reciprocally developed among CURA partners in order to demonstrate culturally responsive mental health practices. And seventh, the project was coordinated by an organisation (CCBR) that is community-based, allowing for a safe and trusted location for the study and all its players. For more information, see: www.communitybasedresearch.ca/ takingcultureseriouslyCURA/.

\section{PAR VALUES: A FRAMEWORK FOR ENTRY}

The use of PAR values as a framework for approaching communities to collaborate on research was generally successful. Our entry strategies served as a prelude to building a true partnership between community participants and the research team. Yet, as in all research involving multiple partners and stakeholders, this process was not as simple as it would appear. Putting values into practice is challenging. For this CURA project, we worked with five different cultural-linguistic communities in two locations. We also partnered with more than 20 local community-based organisations and cultural-linguistic groups. For each new partner or group, a new entry process began, with new and different relationships to be negotiated. 
Through the examples below, we illustrate how the PAR values were used to frame the research entry processes. We discuss the implementation dilemmas and then reflect on the strategies used in various stages of the research entry process.

\section{Empowerment}

In implementing the value of empowerment we used the principle of researching with, not on or for, the culturally diverse communities. This translated into democratic participation of community involvement in all stages of research with voice, choice and control held by communities over research processes and findings. In deciding whether or not to collaborate with one another in a research partnership, researchers and community members addressed several questions. Who are the partners? How will the power be shared among them? What is the focus of the research? How will the knowledge produced be shared and used? The conventional role of researcher (people in control over research processes and results) was challenged and changed into one of collaborator, partner and facilitator of democratic processes.

Similar to what has been noted by Lee (1993) and others in the literature, much of what was encountered in the entry process involved building trust, encouraging ownership and involvement, and overcoming tensions related to negative past experiences with research. We found that implementing the value of empowerment was crucial, but was difficult to achieve, for two reasons:

-it involved a shift in the understanding of the roles of the researchers and the research participants (necessitating ongoing clarifications

- it involved recognition of privileges, stereotypes, racism and power imbalances and the need to build a shared ownership of the research processes and findings (demanding strong facilitation).

\section{Supportive Relationships}

When implementing this value, clear communication and a conscious de-emphasis of professional jargon were important but not sufficient. The powerful role of language (both professional and English) was one of the significant barriers addressed right from the beginning.

The value of supportive relationships was a driving force for us to be truly inclusive, respectful and collegial. In terms of community entry, this was something continuously under negotiation whereby relationships were developed and built over time. People reacted to what was said and how, and talked to each other later - which sometimes helped, sometimes hindered the further development of relationships. We tried to develop and maintain strong, supportive and reciprocal relationships, especially with the community researchers (10 people representing various communities) and the steering committee (12-15 people representing communities, service providers and academics at each site). Just as with other types of relationships, researchercommunity relationships need to be able to demonstrate ongoing trust. 


\section{Social Justice}

Early on in the conceptualisation of our research study, a social change agenda was articulated as its driving force. There were two main reasons for this: to raise community awareness about mental health issues; and to create a more inclusive and effective mental health system. Crucially, however, social justice and action was viewed not only as a potential outcome, but also as an ongoing development throughout. As the entry progressed and relationships developed, participants started to see the research itself as a tool with which communities could take much needed action for change.

\section{Reciprocal Education}

The PAR value of mutual learning throughout the research study was an important selling factor in starting to work with the communities. We promoted the CURA study as an invitation for everybody to create a community of practice where people could learn from each other and where knowledge would be exchanged from and to all involved. The most important aspect when facilitating community meetings was in the asking of questions (not in having all the answers), and in listening to the diverse perspectives and ways of understanding. We tried to incorporate all suggestions and directions coming from the community into the research processes to make them truly collaborative.

\section{Respect for Diversity}

A primary component of the research during the entry process was negotiating the reality of entering a collaborative research project with communities who speak different languages, who have different cultural backgrounds, and who practise different cultural and religious norms and beliefs. At each stage of the entry process, researchers entering the cultural-linguistic communities were evaluated by members of the communities on their actions, assumptions, behaviour and words they used, which often led to reflective consideration of their own cultural norms and values, resulting in an ongoing negotiation between the two. We were actively looking for commonalities across all diverse partners and were appreciating differences in actions and perspectives.

\section{RESEARCH ENTRY STAGES}

In addition to being committed to and explicit about the values of PAR, we went through four distinctive stages of entry and used concrete strategies to engage the ethno-linguistic communities in the research. We would like to briefly describe the four main stages of our entry process: pre-engagement; engagement; assessment, reflection and feedback; and ongoing maintenance (see Lo \& Fung 2003). Thereafter, we critically reflect on the challenges in implementing PAR values. We use our own researchers' field notes as illustrations. 


\section{Pre-engagement Stage}

This was the preparatory stage for the proposal development undertaken before making any official contacts with the cultural communities. According to Lo \& Fung (2003, p. 162), essential to pre-engagement is cultural sensitivity, which 'encompasses attributes such as curiosity, perceptiveness, and respect'. For our entry process, this stage involved respectfully engaging 'gatekeepers' as key informants to help in proposal development and in organising initial site visits with a small number of community members. These pre-meetings with key people in a given community proved to be a helpful first step in connecting with the community itself. It was an opportunity not only for presenting research ideas and inviting communities to participate in research collaboration but also a chance for us to introduce ourselves (and the values we held). In most cases, the initial meeting lasted one to two hours and ended with a clear answer of yes or no to the proposed research collaboration.

Next, a mixed community meeting was organised with leaders from the five selected ethno-linguistic communities in each of the study sites (10-15 community leaders attending each event). Some participants had previously collaborated with research partners and served as brokers of trust between researchers and the communities. The objective of these mixed community meetings was to present the research proposal, to have community leaders subsequently shape the research ideas, to explore ways of working together and to secure preliminary affirmation of community involvement.

These pre-engagement meetings reinforced for us the importance of making initial contacts with an appropriate community gatekeeper, someone who is well connected and well respected within the specific community and who sees the value in collaborative research and who can convince others to join in. In the case of the Punjabi community, not only was he/she able to provide the translation, as needed, but also the necessary interpretations and appropriate arguments. His/ her belief in the importance of this study was a persuasive factor in the community's decision to work with us. (Excerpt from Toronto site)

In order to broaden the involvement of community members and to deepen their engagement, a series of face-toface community site visits were held with members of the five communities in each location. Facilitation was important, as was the language we used (no research jargon), the addressing of challenging issues (such as previous bad research experiences, racism, power imbalances and privileges in research), and an awareness of diversity and our own cultural norms and values. We actively looked for commonalities and appreciated differences among people:

[Flora] borrowed a tunic and headscarf from a co-researcher who had been to India. [Janis] wore a long dress with a scarf she had bought in India. When we arrived at the temple, we arranged our headscarves 
and stepped into the building. The entry hall was filled with people of various ages, all of whom were barefoot. We felt self-conscious about our footwear, but did not know where to leave our shoes. We asked at the front desk for our key contacts, and a man went to find them. Our male contact soon emerged from the room where the religious service was taking place, showed us where to leave our shoes, wash our hands, and then took us into a small room for tea. We used an appropriate greeting in Punjabi, 'Sat-sri-akal'; people responded with big smiles. We were in ... (Excerpt from Waterloo site)

\section{Engagement Stage}

Once funding was approved, a second wave of community visits was held. A series of 10 meetings was organised with the five cultural linguistic communities at the two project sites. In a few cases, additional follow-up meetings were organised to engage more community members.

This engagement stage was the high point of negotiation with community partners, focusing on their involvement, their control, and their benefits and costs. Essential in this stage was to gain mutual understanding of the expected research processes and outcomes, and to clarify roles and responsibilities. Also at this stage we defined the mechanisms for the ongoing community engagement.

Discussions during the site visits were generally lively, if not at times challenging, with community members often 'testing' researchers as to the benefit (and potential harm) of the research to their community (Ochocka \& Janzen 2007). A pressing objective of this project was to learn from the ethno-cultural groups involved as much as possible about their perceptions of mental health/ mental illness, their experiences with services available and, most importantly, their ideas for more appropriate and beneficial support. So community site visits were used to explore these issues and to educate us about how to ask sensitive questions and to listen to diverse ways of understanding. Facilitation of these meetings was aided by our welcoming these challenges, by presenting our commitment to PAR values and by inviting community members to help shape the research agenda through their involvement in the other pre-determined strategies. The following excerpt illustrates various meeting dynamics:

At first, people listened politely. Then they began to ask questions, insisting on needing definitions for terms, parameters for the research, and anticipated outcomes, clearly expecting us to hold ownership of the project. We explained that we have deliberately not defined terms at this point because we want to leave room for definitions that meet each community's needs. With each question, we replied by asking the community to share with us what would make the most sense from their perspective. Our apparent unwillingness to provide answers seemed to be perplexing to participants at first, but once they realized that they had the power to shape the language, definitions and 
parameters of the project for their community, the energy in the room shifted and they took ownership of their participation. (Excerpt from Mandarin-speaking community)

The next step was to establish steering committees, to which each community sent one or two representatives. The main purpose of these steering committees was to serve as a forum for ongoing relationship building, as well as to provide guidelines about project activities. These included participant recruitment, formulation of research methods and tools, data collection and analysis, dissemination strategies, and the development and evaluation of demonstration projects at the two sites. In community-based research projects such as these, the steering committee acts as a sounding board to test ideas before going out into the larger community, and allows the researchers to tailor the research as much as possible to the groups involved (see also Ochocka \& Janzen 2007).

Also at this stage, 10 community researchers were hired from within the 10 cultural-linguistic groups. The bulk of the funding for these researchers was separately raised from $a$ community foundation (the Ontario Trillium Foundation) due to restrictions from the major academic funder. As an essential component of the entry process, these community researchers were hired not only to help with the data collection phase of the project, but also to serve as active voices and as representatives from within their individual communities. Community researchers were not hired on the basis of research skills or experience alone, but also on account of the interpersonal and communication skills they possessed. These 10 community researchers were active and collaborative members of the CURA research team and served as a means of continuous informal information exchange between their respective ethnic communities and the research team.

Thus, the engagement stage for this project was about the development and solidification of the research alliance, the mutual conceptualisation of roles and the establishment of personal rapport.

\section{Assessment, Reflection and Feedback Stage}

The relationships between researchers and community members were tested over the duration of research entry. The assessment, reflection and feedback stage involved daily internal check-ins for the CURA coordination team, and regular checks with individual and group community partners to gather information, reflect on it and adjust entry strategies. Also in this stage we built the structures for the ongoing evaluation and feedback that would occur throughout the research study. This stage emphasised that community entry is indeed an ongoing process; a continuous evaluation of both the implementation of the PAR values and the promises made to communities during the pre- and engagement stages. 
In our CURA project the constant check-ins with steering committee members and community researchers were 'must-do' parts of each bimonthly meeting. The CURA Partnership Group held semi-annual meetings to reflect on entry processes and to provide ongoing feedback to shape them.

This is a very good research centre. When I have problems, I call them and I get private training ... informal training in addition ... whenever you have problems, they solve it with you. A lot of things are new for me, so I've got excellent training and the people are there for me.

(Excerpt from focus group meeting with community researchers)

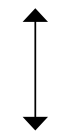

Research Working Groups

- Literature review

- Web Survey

- Key Informant

Interviews

- Case Studies

- Popular Theatre

- Focus Groups

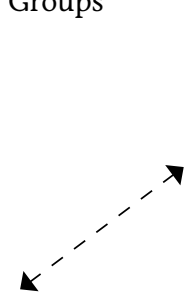

Toronto Somali community
Training
Working Group

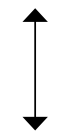

Research Team

Project researchers, student researchers, community researchers

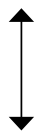

Knowledge Mobilization Working Group
Evaluation Working Group
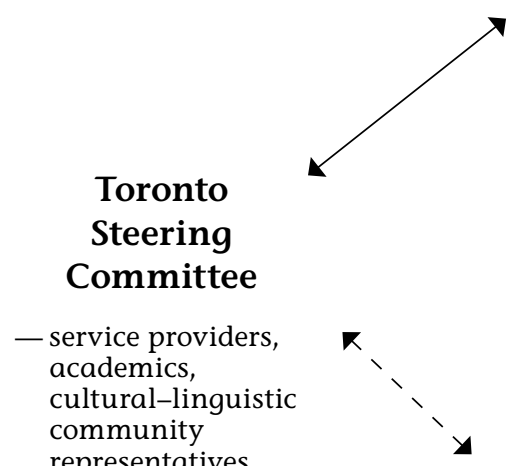

Toronto Polish
community

Toronto

Mandarin

community cultural-linguistic community representatives
Toronto Sikh

Punjabi community
Toronto Latin

American

community

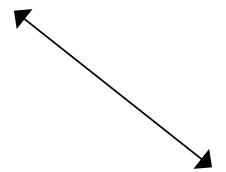

Waterloo

Steering

Committee

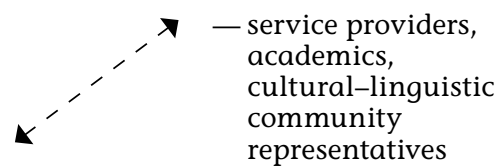

Waterloo Somali
community

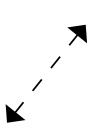

Waterloo

Sikh Punjabi community

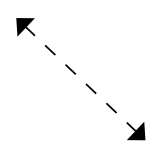

Waterloo Polish community
Waterloo Latin

American

community

\section{Ongoing Maintenance Stage}

This final stage involved developing and shaping sound project structures that would facilitate the equitable participation of all partners in the research and information sharing. These structures enabled open communication and mutual leadership (as well as the entry of new partners throughout the research process). Figure 1 shows the CURA project structure.

There were four working groups with a number of subcommittees. The CURA Partnership Group and two steering committees (at Toronto and Waterloo) provided regular mechanisms for active involvement in the planning and evaluation of all CURA activities. The Knowledge Mobilization Working Group consisted of a mix of diverse 
Table 1: Summary of strategies for each entry stage
CURA partners and was active in providing creative ideas for disseminating CURA news and findings to a variety of audiences (see Jacobson et al. 2007). The Evaluation Working Group was closely engaged in monitoring community engagement and reflecting on CURA activities.

All groups and committees in the CURA structure also served as a means for ongoing reciprocal education. The following excerpt from field notes at the Waterloo site visit with the Somali community emphasises the value of reciprocal education:

This group pointed out that stress and depression are not recognized as 'mental health' issues in their community. To the Somali people, mental health disorders refer only to serious diagnosed illnesses such as schizophrenia. This was important for us to note since it will affect the terminology we use with this community. We were also told that they are reluctant to seek conventional medical care, and will first seek counselling among extended family and religious leaders. (Excerpt from Somali community)

In the maintenance stage we also focused on developing a social aspect to our collaboration. People were invited to participate in gatherings organized by CURA researchers and community groups: for example, CCBR picnics, Christmas parties, trips to conferences, lunches, Chinese New Year celebrations, Punjabi Khalsa, Polish Day, and the Multicultural Festival.

Contained in Table 1 is a summary of the strategies used for each of the entry stages.

\begin{tabular}{l|l}
\hline Entry Stages & Strategies \\
\hline $\begin{array}{l}\text { Pre-engagement } \\
\text { (proposal development) }\end{array}$ & - Meeting with 'gatekeepers' \\
& - Mixed community meeting with \\
& community leaders \\
& -Face-to-face site visits with a small \\
& number of community members \\
\hline Engagement & -Face-to-face site visits with each \\
& community \\
& - Establishment of two steering \\
& committees \\
& - Hiring community researchers \\
\hline Assessment, reflection \& & - Ongoing check-ins with researchers \\
feedback & and community partners \\
& - Collaboration on research (various \\
& working groups) \\
\hline Ongoing maintenance & - Regular communication (at and \\
& between meetings) \\
& - Education focus of the collaboration \\
& - Social focus of the collaboration \\
\hline
\end{tabular}

\section{CHALLENGES IN IMPLEMENTATION}

The four stages of the entry process used various strategies to engage communities in research. The five values of PAR guided all engagement strategies and because of them the community 
entry for this CURA project was generally successful. However, the challenges and tensions around implementing PAR values in the entry process were many. For example, focusing on the value of empowerment was helpful in finding creative ways to share power, information and resources with communities, but was challenging and demanding on a practical level. Questions include: How to build community resonance on an issue under study? How to guarantee community control in decision making when so often researchers are in the 'driver's seat'? How to have an equal collaboration with community groups and organisations when the cost of / availability of resources and funders' expectations are the bottom line? How to facilitate ownership and build trust with many diverse players, and what's truly for them?

Another challenge was ongoing confusion over role clarity throughout the entry process. Making the research process fully collaborative and reciprocal for all involved (contributing and benefiting equally) was full of tensions. The implementation of the value of supportive relationships enabled people to contribute to the process but also challenged them with respectful disagreements and ongoing clarifications, discussions and compromises. It also forced the research partners and participants to consider how supportive they can be and of whom.

We found the implementation of the social justice value in the entry process particularly difficult. Two challenges were observed: one related to realistic expectations about the outcomes of change and the second related to the process of change. In the entry stage community members wanted researchers to make declarations about concrete, tangible and long-term changes in the way systems operate. However, given the nature of any ethically sound research this was not a promise that could be made, as long-term outcomes cannot be pre-determined. This uncertainty was difficult for some partners to live with, particularly for those who had had negative experiences with research projects in the past. Regarding the process of change, the challenge lay in understanding research as intervention. Research from a PAR framework holds the notion of research as social action. Thus, the research process itself provides ample opportunity for some level of social change and action from within the communities involved to occur. Although this is not a guaranteed outcome for all PAR research projects, in this case there were indications right from the entry stage that some level of social change was taking place from within the cultural-linguistic communities as they became more actively involved in the research project. Part of this was due to simply opening up the public dialogue about mental health and mental illness within the communities, which allowed for awareness raising, stigma reduction and community capacity building around support to start taking place. As the research process continued, this momentum towards change from within the communities continued as well. 
The implementation of reciprocal education built trust throughout, but it again created a situation of unpredictability and open-endedness within the project planning, which some communities had difficulty with. Being true to the value of reciprocal education meant that everyone was forced to challenge at some point in the entry process his or her own assumptions and taken-for-granted knowledge. We, of course, learned a lot about the specifics of ethno-cultural communities and their experiential realities, but we also learned about our own assumptions.

And finally, given that this project involved the participation of many cultural-linguistic groups, there were some challenges encountered when implementing the value of respect for diversity. First was the simple challenge of becoming as familiar as possible with the cultural norms and values of each of the five cultural-linguistic groups involved and incorporating this into the research as far as possible. Second, once the groups were gathered all together, was the challenge of agreeing on a common purpose that made sense to everyone and, in some cases, agreeing to disagree when necessary. Third, there was a challenge of moving the process forward without being 'caught' in individual/ group differences. And finally, there was the challenge of the background context (for example, previous negative research experience, the existence and previous experience of racism and discrimination, a lack of trust in the possibility of building new relationships that could be free of power imbalances and manipulation). Although challenging, this value was the most significant for the entry process, as it was crucial for forming strong, long-term relationships that would facilitate the research over the five years to come.

\section{CONCLUSION}

In this article, we have presented a framework for community entry when conducting research on sensitive topics and have illustrated how this framework was put into practice in a Community University Research Alliance in Ontario, Canada. This article emphasises three points about research entry. First, that research entry is not only the effort at the beginning of the research project but is ongoing (before and throughout all phases of the research). Second, that successful research entry is about building and maintaining strong and reciprocal relationships between researchers and community members. Third, that research entry employs both PAR values and concrete engagement strategies (Lo \& Fung 2003; Ochocka \& Janzen 2007).

In presenting this case study as an example of a successful approach to research entry, we want to emphasise how important the combination of PAR values and engagement strategies is in developing reciprocal and action-oriented relationships between researchers and community members. PAR values guide researchers' strategies (and their implementation) for the recruitment of study participants but also for building lasting 
partnerships that help in effective knowledge production as well as knowledge mobilisation for future change. Given the richness of experience that has been gained through working with this CURA project, we believe other community-based research could benefit from this presented framework.

There are some potential challenges in using a PAR framework in the entry process. These include previous negative experience with community-based research, working with different languages, cultures or religious backgrounds, choosing the right gatekeepers, and finding a balance between collective and individual ownership of research outcomes. It seems to us that successful entry, which focuses on developing and maintaining trusted relationships, depends on an ongoing effort to maintain transparency and inclusivity for all involved, and on focusing on commonalities. It also requires a vision to use research for desirable change. Maintaining a strong commitment to the implementation of the PAR values in each of the various engagement strategies results in respectful, strong and mutually beneficial partnerships.

Beyond challenges, we believe that there are two main dilemmas to research entry using PAR values. First is a strong pressure for researchers to deliver. Communities engaged in research are constantly evaluating researchers and project coordinators according to the promises made in the preengagement and engagement stages. Sometimes the expectations are too ambitious. Second is the ability to play various roles with different community partners at the different stages of the research entry process. Often researchers need to be inspiring leaders or initiators for action, but sometimes they need to play a follower/ junior role allowing communities to lead and coordinate activities. Constant negotiation and renegotiation of relationships are needed with all players. The development of real, reciprocal and actionoriented relationships between researchers and communities are the only way to make research relevant and research entry possible and successful.

\section{REFERENCES}

Babbie, E 1998, The practice of social research, Wadsworth Publishing Company, Belmont, CA.

Chamberlin, J 1997, 'A working definition of empowerment', Psychiatric Rehabilitation Journal, vol. 20, no. 4, pp. 43-46.

Heron, J \& Reason, P 1997, 'A participatory inquiry paradigm', Qualitative Inquiry, vol. 3, no. 3, pp. 274-94.

Israel, B, Schulz, A, Parker, E \& Becker, A 1998, 'Review of communitybased research', Annual Review of Public Health, vol. 19, pp. 173-202.

Jacobson, N, Ochocka, J, Wise J, Janzen, R \& the 'Taking Culture Seriously' Partners 2007, 'Inspiring knowledge mobilization through a communications policy: The case of a Community University Research Alliance', Progress in Community Health Partnerships: Research, Education and Action, vol. 1, no. 1, pp. 99-104.

Janzen, R, Ochocka, J \& the 'Taking Culture Seriously' Partners 2006, 
'The road toward cultural empowerment: An invitation to inclusion', in D Zinda (ed.), Navigating multiculturalism: Negotiating change, Cambridge Scholar's Press, Newcastle, UK, pp. 58-76.

Janzen, R, Ochocka, J, Jacobson, N, Maiter, S, Simich, L, Westhues, A, Fleras, A \& the 'Taking Culture Seriously' Partners 2010, 'Synthesizing culture and power in community mental health: An emerging framework', Canadian Journal of Community Mental Health, vol. 29, no. 1, pp. 51-67.

Kemmis, S \& McTaggart, R 2005, 'Participatory action research: Communicative action and the public sphere', in N Denzin \& Y Lincoln (eds), The Sage handbook of qualitative research, 3rd edn, Sage, Thousand Oaks, CA, p. xix, pp. 559-603.

Lee, R 1993, Doing research on sensitive topics, Sage, London.

Lindsay, J 2005, 'Getting the numbers: The unacknowledged work in recruiting for survey research', Field Methods, vol. 17, no. 1, pp. 119-28.

Lo, H \& Fung, K 2003, 'Culturally competent psychotherapy', Canadian Journal of Psychiatry, vol. 48, no. 3, pp. 161-70.

Minkler, M 2004, 'Ethical challenges for the "outside" researcher in community-based participatory research', Health Education \& Behavior, vol. 31, no. 6, pp. 684-97.

Nelson, G, Janzen, R, Ochocka, J \& Trainor, J 2010, 'Participatory action research and evaluation with mental health self-help groups and organizations: A theoretical framework', in L Brown \& S Wituk (eds), Mental health self-help: Consumer and family initiatives, Springer, New York.

Nelson, G, Lord, J \& Ochocka, J 2001, 'Empowerment and mental health in community: Narratives of psychiatric consumer/survivors', Journal of Community \& Applied Social Psychology, vol. 11, no. 2, pp. 125-42.

Nelson, G, Ochocka, J, Griffin, K, \& Lord, J 1998, “"Nothing about me, without me": Participatory action research with self-help/mutual aid organizations for psychiatric consumer/survivors', American Journal of Community Psychology, vol. 26, no. 6, pp. 881-912.

Nelson, G, Ochocka, J, Janzen, R, Trainor, J \& Lauzon, S 2004, 'A comprehensive evaluation approach for mental health consumer-run organizations: Values, conceptualization, design, and action', Canadian Journal of Program Evaluation, vol. 19, no. 3, pp. 29-53.

Ochocka, J 2007, 'Working with diverse communities towards social change: A community university partnership in Canada using a participatory action research approach', in A Bokszczanin (ed.), Social change in solidarity: Community psychology perspectives and approaches, University of Opole Press, Opole, Poland, pp. 76-83.

Ochocka, J \& Janzen R 2007, ‘Blending commitment, passion and structure: Engaging cultural linguistic communities in collaborative research', in A Williamson \& $\mathrm{R}$ DeSouza (eds), Researching with communities: Grounded perspectives on engaging communities in research, Muddycreek Press, Auckland, New Zealand.

Ochocka, J, Nelson, G \& Janzen, R 2005, 'Moving forward: Negotiating self and external circumstances in recovery', Psychiatric Rehabilitation Journal, vol. 28, no. 4, pp. 315-22.

Parrado, E, McQuiston, C \& Flippen, C 2005, 'Participatory survey research: Integrating community collaboration and quantitative methods for the study of gender and HIV risks among Hispanic migrants', 
Sociological Methods \& Research, no. 34, no. 2, pp. 204-39.

Rappaport, J 1981, 'In praise of paradox: A social policy of empowerment over prevention', American Journal of Community Psychology, vol. 9, no. 1, pp. $1-25$.

Reason, P 2006, 'Choice and quality in action research practice', Journal of Management Inquiry, vol. 15, no. 2, pp. 187-203.

Sadavoy, J, Meier, R \& Ong, A 2004, 'Barriers to access to mental health services for ethnic seniors: The Toronto study', Canadian Journal of Psychiatry, vol. 49, no. 3, pp. 192-99.

Schensul, J 2009, 'Community, culture and sustainability in multilevel dynamic systems intervention science', American Journal of Community Psychology, vol. 43, no. 3-4, pp. 241-56.

Simich, L, Maiter, S \& Ochocka, J 2009, 'From social liminality to cultural negotiations: Transformative processes in immigrant mental wellbeing', Anthropology \& Medicine, vol. 16, no. 3, pp. 253-66.

Simich, L, Maiter, S, Moorlag, E \& Ochocka, J 2009, “"Taking Culture Seriously": Ethno linguistic community perspectives on mental health', Psychiatric Rehabilitation Journal, vol. 32, no. 3, pp. 208-14.

Sixsmith, J, Boneham, M \& Goldring, J 2003, 'Accessing the community: Gaining insider perspectives from the outside', Qualitative Health Research, vol. 13 , no. 4 , pp. $578-89$.

Stringer, E 2007, Action research, 3rd edn, Sage, Thousand Oaks, CA.

Tareen, E \& Omar, M 1997, 'Community entry: An essential component of participation', Health Manpower Management, vol. 23. no. 3, pp. 97-99.

Wenger, E, McDermott, R \& Snyder, W 2002, Cultivating communities of practice: A guide to managing knowledge, Harvard Business School Press, Boston, MA.

Zimmerman, M 1995, 'Psychological empowerment: Issues and illustrations', American Journal of Community Psychology, vol. 23, no. 5, pp. 581-99. 